\title{
Componentes funcionais da teoria de Peplau e sua confluência com o referencial de grupo*
}

\author{
Analysis of the Functional components of the Peplau's theory and its confluence with the group \\ reference \\ Componentes funcionales de la teoría de Peplau y su confluencia con el referencial de grupo
}

\section{Leila Memória Paiva Moraes ${ }^{1}$, Marcos Venícios de Oliveira Lopes², Violante Augusta Batista Braga ${ }^{3}$}

\begin{abstract}
RESUMO
As teorias de enfermagem precisam ser analisadas para que possamos acessar o real significado das idéias explicitadas pelas teóricas e, com isso, possibilitar que os enfermeiros as considerem e as incorporem na prática profissional. Este estudo objetivou analisar os componentes funcionais da teoria de Hildegard Elizabeth Peplau, com base no modelo proposto por Meleis e sua confluência com o referencial de grupo utilizado no trabalho com dependentes químicos. A construção deste estudo proporcionou, não apenas conhecer os componentes funcionais desta teoria, onde os mesmos foram identificados, mas, principalmente, sua adequação e utilização a grupos no âmbito da cotidianidade da prática de cuidar do dependente químico.
\end{abstract}

DESCRITORES: Teoria de enfermagem; Saúde mental; Relações interpessoais; Modelos de enfermagem

\begin{abstract}
To facilitate nurses' application of nursing theories, theories must be analyzed and disseminated. This study aimed to analyze the functional components of Paplau's Theory of Nursing among chemical dependent participants. The analysis of the theory was based on Meleis's model. The results of this analysis provided more than an understanding of the functional components of this theory; the results included the appropriateness and utilization of Paplau's theory to provide care to dependent chemical clients.

keywords: Nursing theory; Mental health; Interpersonal relations; Models, nursing
\end{abstract}

\section{RESUMEN}

Las teorías de enfermería necesitan ser analizadas para que podamos acceder al real significado de las ideas explicitadas por las teóricas y, con eso, posibilitar que los enfermeros las consideren e incorporen en su práctica profesional. Este estudio tuvo como objetivo analizar los componentes funcionales de la teoría de Hildegard Elizabeth Peplau, con base en el modelo propuesto por Meleis y su confluencia con el referencial de grupo utilizado en el trabajo con dependientes químicos. La construcción de este estudio permitió, no sólo conocer los componentes funcionales de esta teoría, donde los mismos fueron identificados, sino, principalmente, su adecuación y utilización a grupos en el ámbito cotidiano de la práctica de cuidar del dependiente químico.

DESCRIPTORES: Teoría de enfermería, Salud mental; Relaciones interpersonales; Modelos de enfermería

\footnotetext{
*Estudo realizado na Disciplina de Análise Crítica das Teorias de Enfermagem, do Curso de Doutorado em Enfermagem, Programa de Pós-Graduação em Enfermagem, da Universidade Federal do Ceará-UFC-Fortaleza (CE), Brasil. .

${ }^{1}$ Mestre em Enfermagem. Doutoranda em Enfermagem da Universidade Federal do Ceará-UFC-Fortaleza (CE), Brasil; Bolsista da FUNCAP - Fundação Cearense de Apoio ao Desenvolvimento Científico e Tecnológico (FUNCAP).

${ }^{2}$ Doutor em Enfermagem. Professor Adjunto do Departamento de Enfermagem da Universidade Federal do Ceará -UFC- Fortaleza (CE), Brasil.

${ }^{3}$ Doutora em Enfermagem. Professora Adjunto do Departamento de Enfermagem da Universidade Federal do Ceará-UFC- Fortaleza (CE), Brasil..
} 


\section{INTRODUÇÃO}

O trabalho de grupo é algo que tem crescido na prática de enfermagem em saúde mental, principalmente se o mesmo tem por objetivo a ação terapêutica. É importante abordarmos, de forma grupal, pessoas com sofrimento psíquico ou que apresentem grandes potencialidades para desenvolver uma doença mental, assim como, os dependentes químicos. Essa forma de cuidar favorece a interação e integração de seus participantes, contribuindo, ainda, para o processo de aprendizagem e de crescimento pessoal.

Propomos que esse processo de aprendizagem seja baseado, não só, na abordagem grupal, mas que seja guiado por uma teoria de enfermagem, especialmente de base interacionista, como a teoria das relações interpessoais em enfermagem de Hildegard Peplau.

Em se tratando do cuidado a dependentes químicos o enfermeiro é um dos principais atores que integra a equipe interdisciplinar na abordagem a este tipo de cliente. Esse profissional deverá ter, em sua formação, condições técnicas de exercer o papel de psicoterapeuta, podendo trabalhar com evidencias de que as dificuldades da vida do cliente são possíveis determinantes do processo saúdedoença mental, e estas devem ser identificadas e trabalhadas com a ajuda do enfermeiro ${ }^{(1)}$.

Por ser o dependente químico um cliente que demanda um intenso esforço próprio na busca da recuperação e prevenção da recaída do uso e ou abuso de drogas, a enfermagem deve atuar no sentido de fortalecer esse cliente, auxiliando-o a mover-se em direção ao pleno desenvolvimento de seu potencial. Isto deve contribuir para que o mesmo possa viver produtivamente na família e comunidade, sem o abuso de drogas. As metas e ações de enfermagem deverão ajudar esse cliente a vencer os obstáculos da toxicomania, permanecendo nele um desejo cada vez mais forte de continuar em abstinência.

Como cuidar de dependentes químicos numa abordagem grupal demanda, entre várias outras coisas, habilidades em técnicas interpessoais de comunicação e relacionamento terapêutico e técnicas de manejo grupal, nos propusemos a iniciar um estudo de análise reflexiva sobre a teoria da relação interpessoal em enfermagem de Hildegard Peplau, a qual objetiva o cuidado humano centrado no processo interpessoal entre enfermeiro e cliente.

A teoria de Peplau foi baseada no modelo psicodinâmico, o qual visa apreender as necessidades do cliente, identificando dificuldades e procurando ajudá-lo a superar, através de um cuidado de enfermagem individualizado. É uma teoria com forte pertinência à área de Enfermagem em Saúde Mental, pois o ser humano deve ser visto como um todo, e o enfermeiro necessita do conhecimento de outras ciências para que possa conhecer e intervir junto a esse cliente. O papel maior do enfermeiro é estabelecer o processo de comunicação e relacionamento terapêutico como objetivo principal do cuidado humano, estimulando o enfrentamento das dificuldades ou problemas e o desejo de permanecer saudável ${ }^{(2-3)}$.

Diante do exposto, temos como objetivo do nosso estudo analisar os componentes funcionais da teoria de Hildegard Peplau e sua confluência com o referencial de grupo utilizado no trabalho com dependentes químicos.

\section{Critérios de análise teórica}

Este estudo, teórico-reflexivo da Teoria de Hildegard Elizabeth Peplau, buscou analisar sua adaptação à assistência e pesquisa com grupos terapêuticos de dependentes químicos. Para isso utilizamos alguns dos critérios de análise de teorias propostos por Afaf Meleis $^{(4)}$.

O modelo de análise de Meleis é dividido nos seguintes segmentos: descrição, análise, crítica, teste e apoio da teoria ${ }^{(4)}$. Nosso foco se limitou ao segmento descrição da teoria, o qual é dividido em componentes estruturais e funcionais, no estudo em particular nos delimitamos aos componentes funcionais. Essa etapa de análise é responsável por identificar conceitos pertinentes às idéias centrais de uma teoria e, no estudo em particular, a congruência desses conceitos com a utilização da teoria de Peplau a grupos de pessoas dependentes químicas.

Entre os componentes funcionais que estão inseridos na descrição, destacamos: o foco, o cliente, a enfermagem, a saúde, a interação enfermeiro/cliente, o ambiente, os problemas de enfermagem e as intervenções de enfermagem ${ }^{(4)}$.

Ressaltamos as seguintes questões centrais descritas pelo modelo de análise de Meleis que permearam o estudo dos componentes funcionais da teoria: A teoria identifica seu foco com o cliente, a família, a comunidade e a sociedade? Qual a definição que a teoria oferece de enfermagem, cliente, saúde, problemas de enfermagem, relacionamento enfermeiro-paciente? São definições explícitas ou implícitas? A teoria oferece uma idéia clara sobre os problemas de enfermagem? A teoria oferece algum insight no que concerne a intervenção de enfermagem? ${ }^{(4)}$

A identificação desses componentes funcionais subsidiou nossa reflexão no que se refere à utilização da teoria de Peplau a grupos de dependentes químicos. A estratégia utilizada buscou desenvolver um diálogo crítico-reflexivo entre teóricos de grupo, explorando a coerência de seus pensamentos, sua clara identificação da Teoria das Relações Interpessoais em Enfermagem ${ }^{(2)}$, e estabelecendo-se associações e identificações entre os 
componentes funcionais do modelo de Meleis ${ }^{(4)}$.

\section{Grupo terapêutico e a assistência de enfermagem}

O trabalho com grupos se constitui um dos principais recursos terapêuticos nos mais diferentes contextos de assistência à saúde. Essas terapias grupais têm crescido na área da enfermagem e a assistência em Saúde Mental é uma das grandes responsáveis por esse crescimento. No Brasil, a terapia de grupo, em diferentes abordagens, é praticada por grande número de profissionais de áreas diversas, mas, principalmente, pelos profissionais de Saúde Mental ${ }^{(5)}$.

Historicamente, a terapia de grupo surgiu em 1905, quando grupos formados por pessoas portadoras de tuberculose aprendiam sobre sua doença e recebiam apoio emocional. Mas, somente à época da Segunda Guerra Mundial, torna-se uma intervenção utilizada para o tratamento de pessoas com diagnóstico de doença mental ${ }^{(6)}$.

De fato, a psicoterapia de grupo originou-se nos anos vinte, do século passado, com base na aplicação das teorias de grupo de várias escolas psicoterápicas - Freudiana, Sullivaniana, Rogeriana, Bernieniano. Os terapeutas que tinham sido treinados em uma dessas teorias de psicoterapia começaram a aplicar a estrutura de tratamento deles em grupos, passando a buscar conceitos para desenvolver técnicas específicas ${ }^{(7)}$.

A abordagem grupal é um modo de assistir em Saúde Mental, em que o enfermeiro se torna um profissional autônomo, conhecedor dos benefícios à natureza humana, bem como dos seus limites teóricos e práticos ${ }^{(5)}$.

Grupo não é um mero ajuntamento de pessoas, mas, um sistema identificável, composto de três ou mais indivíduos que se engajam em tarefas a fim de atingirem um objetivo comum. Seus membros devem relacionarse uns com os outros em torno de tarefas e objetivos ${ }^{(6)}$. Deve ser visto como sendo um conjunto de indivíduos que são, até certo ponto, interdependentes ${ }^{(7)}$.

O grupo terapêutico visa o tratamento de pessoas, sendo administrado por um 'terapeuta' com os objetivos de 'insight' e/ou mudança de comportamento. Dependendo da orientação teórica do terapeuta, o grupo pode focalizar o intrapessoal e/ou problemas interpessoais do cliente. Deve ser visualizado como um microcosmo social no qual os clientes demonstram seus sentimentos problemáticos e comportamentos, podendo vivenciar experiências emocionais corretivas de ser respondido diferentemente. O terapeuta assume um papel parental, ajudando os integrantes a aprender modos novos de enfrentamento ${ }^{(7)}$.

A literatura específica sobre grupos terapêuticos desenvolvidos por enfermeiro ainda é predominantemente internacional ${ }^{(8)}$, sendo reduzido, também, o número de publicações que contenham a descrição desta prática com fundamentação teórica consistente ${ }^{(5)}$.

\section{A Teoria das Relações Interpessoais em Enfermagem de Hildegard Elizabeth Peplau}

Hildegard Elizabeth Peplau nasceu em $1^{\circ}$ de setembro de 1909, em Reading, na Pensilvânia e veio a falecer em 17 de março de 1999, aos 89 anos, em sua casa em Sherman Oaks, Califórnia, após uma trajetória profissional que influenciou a enfermagem a ponto de ela ser considerada a "enfermeira do século" e "mãe da enfermagem psiquiátrica" por ter sido pioneira no desenvolvimento da teoria e prática de Psiquiatria e Saúde $\operatorname{Mental}^{(9)}$

As idéias de Peplau têm sido incorporadas em outras práticas profissionais de saúde e sua teoria foi introduzida há aproximadamente quarenta anos, época em que os clientes não eram participantes ativos do cuidado que lhes era prestado ${ }^{(10)}$.

Essa teórica foi introdutora do relacionamento terapêutico enfermeiro-cliente na enfermagem, passando grande parte de sua vida profissional dedicando-se a trabalhos na área da Enfermagem Psiquiátrica. Publicou sua obra Interpesonal Relations in Nursing, em 1952, sua teoria original, a qual foi resultado de seu doutorado em Enfermagem Psiquiátrica ${ }^{(1)}$. A teoria de Peplau foi embasada em conhecimentos das ciências do comportamento, recebendo influências de grandes pensadores da área de psiquiatria, tais como Percival Simonds, Harry Sullivan, Abraham Maslow e Neal Miller ${ }^{(11)}$.

Para construção da teoria Peplau seguiu a vertente das teorias orientadas para interação ${ }^{(12)}$. Além de ser uma teoria interacionista, também se insere no grupo das teorias explicativas, pois explica como a enfermagem é um processo interpessoal relacionando as causas e efeitos da interação, sendo uma abstração sistemática da realidade que pode contribuir para fundamentar a prática na enfermagem ${ }^{(13)}$.

A teoria resume duas condições de interação que são essenciais à saúde: as demandas fisiológicas de um organismo humano que exigem a manipulação das condições materiais em benefício do bem - estar de um indivíduo ou grupo e as condições interpessoais, que são individuais e sociais, e que satisfazem as necessidades da personalidade e permitem a expressão e uso das capacidades de forma produtiva ${ }^{(2)}$.

Em se tratando de cuidados aos dependentes químicos, espera-se que uma assistência grupal a essas pessoas seja um momento de aprendizagem para ambos, enfermeiro e cliente, principalmente para a pessoa do dependente químico que, durante o seu tratamento, convive com a oportunidade de amadurecimento e aprendizagem diante das situações conflitantes e 
ameaçadoras que são resultantes do uso de drogas psicoativas. Assim, busca-se na teoria de Peplau um caminho para a resolução dos problemas do dependente químico, onde esses poderão canalizar suas capacidades para a produção de mudanças que influenciarão em suas vidas de forma positiva.

No processo de relação do enfermeiro com o cliente, a teórica ressalta quatro fases de atuação no processo de enfermagem que deverá ser educativo e terapêutico: orientação, identificação, exploração e resolução, cada fase se caracteriza por papéis ou funções desempenhadas pelo enfermeiro ou cliente à medida que os mesmos aprendem a trabalhar conjuntamente para resolver suas dificuldades $^{(2)}$. Essas fases interrelacionam-se e variam quanto a duração temporal à medida que o processo evolui para o encontro de uma solução, apresentando momentos sobrepostos. A seguir, descreveremos individualmente cada uma dessas fases, buscando sua confluência com a abordagem grupal.

$\mathrm{Na}$ fase de orientação, o enfermeiro e a pessoa/ família/grupo têm o primeiro contato, passando a identificar e compreender as necessidades do problema existente. Durante a orientação às atitudes do cliente e do enfermeiro são influenciadoras sobre o dar ou receber ajuda, onde os participantes do processo interpessoal apresentam partes igualmente importantes na interação terapêutica ${ }^{(14)}$.

Essa fase inicial mostra-se bastante relevante para um grupo realizado com clientes dependentes químicos, pois cada participante expressa suas dificuldades individuais, as quais acabam muitas vezes sendo parecidas com as dos outros participantes do grupo, podendo haver um amadurecimento grupal e uma melhor compreensão da vivência de ser um dependente químico.

Durante a fase de identificação a pessoa seleciona aquelas pessoas que possam ajudá-la, como no caso o enfermeiro ou algum membro do grupo ${ }^{(2)}$.

Se há relacionamento interpessoal satisfatório, nesta fase os participantes do grupo podem começar a ter a sensação de ser capaz de lidar com os seus problemas. Essa transformação diminui os sentimentos de desamparo, motivando-os para o enfrentamento de suas necessidades ${ }^{(2)}$.

A fase de exploração ocorre após o cliente identificar o enfermeiro como sendo aquele que reconhece e compreende as relações interpessoais da situação, passando a explorar todos os serviços que lhe são oferecidos ${ }^{(2)}$.

Quanto ao dependente químico, ele se percebe como a parte integrante mais importante do ambiente provedor de cuidados a saúde, pois essa pessoa deve ser consciente da necessidade de sua plena adesão ao tratamento, ficando atento a todas as formas de ajuda e de informações que possam contribuir com seu processo de superação das dificuldades e de atendimento às necessidades afetadas. É nesse momento que um grupo de pessoas dependentes químicas passa a exigir mais do profissional que está na posição de terapeuta. Ao enfermeiro cabe proporcionar um clima emocional terapêutico, ajudando-o a responder questões individuais e explorar todos os caminhos que conduzam a um comportamento saudável.

$\mathrm{Na}$ fase de resolução espera-se que as necessidades do cliente já tenham sido satisfeitas, começando a se desfazer o elo entre enfermeiro e cliente. Nessa fase o cliente apresenta-se pronto para voltar para casa, abandonando-se, assim, os antigos laços e dependências, implicando na liberação gradual da identificação com as pessoas que tem proporcionado assistência e a criação e fortalecimento da capacidade para agir por si mesmo(2).

Análise dos componentes funcionais da teoria de Peplau

A teoria identifica seu foco com o cliente, familia, comunidade, sociedade?

O foco da teoria é a relação interpessoal, apresentando como objetivo central a busca de uma nova maneira de agir em enfermagem, a partir de certas indagações como: o que é enfermagem? Como agem os enfermeiros? Em que constitui o saber da enfermagem? Essa forma de agir é encarada como sendo uma nova maneira de cuidar, pois, a partir do conhecimento do que seja realmente enfermagem, torna-se mais fácil construir a prática ${ }^{(2)}$.

Como o foco da teoria de Peplau são as relações interpessoais, fatores como a cultura, valores, crenças, expectativas do cliente devem ser levadas em consideração, tendo em vista a participação do mesmo como sujeito ativo do processo.

A teoria, em sua essência, busca trabalhar o relacionamento interpessoal entre enfermeiro/cliente como forma para se chegar à resolução dos problemas que afetam o cliente, família, comunidade ou sociedade.

Qual a definição que a teoria oferece à enfermagem, cliente, saúde, problemas de enfermagem, relacionamento enfermeiro-paciente? São definições explícitas ou implícitas?

Nessa teoria, a enfermagem é definida como sendo um processo significante, terapêutico e interpessoal pelo fato de envolver interação entre duas ou mais pessoas, com meta comum, tendo como funções cooperar com outros processos humanos que tornam a saúde possível a pessoas e comunidades. É uma relação entre um indivíduo que está doente ou necessitado de serviços de saúde e um enfermeiro preparado para conhecer e responder às necessidades de assistência ao cliente. Esta meta proporciona o incentivo ao processo terapêutico, onde os envolvidos nessa relação, enfermeiro e cliente aprendem e crescem como resultado da interação ${ }^{(2)}$.

De acordo com a teoria, enfermagem e educação 
não são coisas diferentes, pois a enfermagem deve ser visualizada como uma força de amadurecimento e um instrumento educativo que aspira fomentar o progresso da personalidade na direção de uma vida criativa, construtiva, produtiva, pessoal e comunitária, onde tanto a enfermagem quanto a pessoa envolvida na ação interpessoal constituem uma experiência de aprendizagem do $\mathrm{eu}^{(2,12)}$.

O cliente é percebido como uma estrutura biológica, espiritual, social e psicológica única, que tem suas peculiaridades e reage de forma singular. A teoria refere que uma família, um grupo ou uma comunidade pode ser coletivamente definido como o paciente ${ }^{(2)}$.

Assim, visualizamos uma congruência da definição de cliente/indivíduo com o cliente/grupo, pois um grupo é uma estrutura única, apresentando suas particularidades e reagindo também de forma singular. Um grupo composto por dependentes químicos vai ser sempre diferente de outro grupo, mesmo que de dependentes químicos.

Já a definição de saúde apresenta-se como uma qualidade dinâmica, que permite a pessoa a experiência potencial de bem-estar físico e social e que confere a esse indivíduo a oportunidade de viver bem e em harmonia com os demais ${ }^{(2)}$.

Os problemas de enfermagem são visualizados como sendo algo que abale a estrutura biológica-psicológicaespiritual e sociológica das pessoas. As variáveis nas situações de enfermagem são: as necessidades, a frustração, o conflito e a ansiedade ${ }^{(2,12,14)}$. O enfermeiro e o cliente (grupo ou família) passam a trabalhar a fim de identificar o problema existente, diminuindo, assim, a tensão, a ansiedade, o medo e as necessidades diversas. Ao diminuirmos esses sentimentos e necessidades estamos prevenindo problemas futuros. Essas possíveis situações estressantes são identificadas através de interações terapêuticas.

Sobre as interações terapêuticas oriundas do processo de relação interpessoal ou relacionamento enfermeirocliente, Peplau conceitua como sendo aquela em que duas pessoas chegam a se conhecer suficientemente para enfrentar os problemas que surjam de forma cooperativa. Para que a dinâmica da relação interpessoal possa acontecer é necessária que as ações do enfermeiro sejam destinadas às pessoas que necessitem de cuidados, de tal modo, que essas possam refletir na comunidade em que vivem.

As definições presentes nos componentes funcionais da teoria, descritas anteriormente, são expressas de formas explícitas, claras e de fácil entendimento. Todavia, a teoria não deixa claro seu conceito de ambiente ficando implícito que o ambiente pode ser o espaço onde se processa a interação entre o enfermeiro e cliente.

\section{A teoria oferece uma idéia clara sobre os problemas de enfermagem?}

A teoria refere que o enfermeiro e cliente devem, inicialmente, esclarecer o problema, explorando expectativas e metas, para que, posteriormente, sejam decididos os planos adequados a fim de melhorar o estado de saúde do cliente, sendo alcançado os objetivos do mesmo.

Há necessidade de uma exploração recíproca da dificuldade do cliente e de sua relação com o enfermeiro a fim de buscar a identificação dos problemas e as possíveis soluções ${ }^{(2)}$. E dessa maneira que ocorre em um grupo, onde, inicialmente se da a identificação entre clientes e terapeutas e, posteriormente, ambos partem para o esclarecimento dos problemas, expectativas e metas dos membros do grupo. Após isso, o terapeuta, com a participação do próprio grupo, parte para possíveis intervenções.

Os problemas de enfermagem aparecem de forma implícitas na teoria, pois eles mostram-se apenas como fatores pessoais, incluindo as necessidades, frustrações, conflito e ansiedade. O processo de enfermagem pode visualizar o cliente de modo coletivo, levando em conta o ambiente como um todo ${ }^{(2)}$.

Em um grupo busca-se a resolução para esses fatores pessoais, os quais podem guardar estreita relação uns com os outros, considerando-se tratar de um grupo onde a clientela apresenta a mesma problemática.

\section{A teoria oferece algum "insight" no que concerne à intervenção de enfermagem?}

De acordo com os parâmetros da enfermagem atual, bem como da teoria de Peplau, a intervenção de enfermagem realiza-se através de um plano de assistência individualizado adequado para melhorar o estado de saúde do cliente, grupo ou comunidade.

Os enfermeiros ajudam e cuidam das pessoas, não as manipulam; pretendem auxiliar indivíduos e comunidades estabelecendo algumas condições para satisfazer às suas necessidades ${ }^{(2)}$. Isto nos deve servir de alerta, a fim de que tenhamos uma postura de facilitadoras do cuidado, identificando, clarificando e traçando objetivos e ações que atendam as situações que requerem nossa atenção.

Percebemos que existem semelhanças básicas entre o processo de enfermagem e as fases interpessoais da teoria de Peplau. Tanto as fases, quanto o processo de enfermagem são seqüenciais e focalizam interações terapêuticas, utilizando técnicas de resolução de problemas, nas quais colaboram enfermeiro e cliente, com o propósito final de satisfazer às necessidades pelas intervenções propostas. Esses dois momentos vão do geral para o específico, incluindo, observação, comunicação e registro, como instrumentos básicos utilizados pelo enfermeiro.

\section{CONSIDERAÇÕES FINAIS}

Ao analisar a teoria das relações interpessoais verificou- 
se que, apesar da mesma ter sido proposta inicialmente quando a enfermagem tinha sua prática voltada mais especificamente para a assistência individual, a autora direciona o trabalho com tríades ou relacionamento com diversas pessoas, criando, assim, uma perspectiva diferente para o relacionamento interpessoal em enfermagem.

Nas fases da teoria percebemos congruência com as fases do desenvolvimento de um grupo, portanto a teoria é aplicável a essa clientela, pois há utilidade nas etapas do relacionamento interpessoal, podendo direcioná-las ao trabalho de grupo da enfermagem na assistência ao dependente químico.

O estudo propiciou a aplicação de parte do Modelo de análise de Meleis, ou seja, a descrição dos componentes funcionais da teoria, se apresentado como um direcionamento eficaz para que o pesquisador conheça e avalie a adequação de um trabalho baseado em teorias.

Os componentes funcionais do modelo buscaram uma identificação mais precisa de elementos da teoria de Hildegard Peplau, tendo como foco, cliente, ambiente, enfermagem, saúde, interação enfermeiro e cliente, problema de enfermagem e terapêutica de enfermagem. Através destes conceitos tivemos uma visão maior das idéias da teoria da enfermeira psiquiátrica e sua possível aplicação para com grupos terapêuticos.

\section{REFERÊNCIAS}

1. Stefanelli MC. Comunicação com paciente: teoria e ensino. 2a ed. São Paulo: Robe; 1993.

2. Peplau HE. Relaciones interpersonales en enfermería: un marco de referencia conceptual para la enfermería psicodinâmica. Barcelona: Masson-Salvat; 1993.
3. Almeida VCF, Lopes MVO, Damasceno MMC. Teoria das relações interpessoais de Peplau: Análise fundamentada em Barnum. Rev Esc Enfermagem USP. 2005; 39(2):202-10.

4. Meleis AI. Theoretical nursing: development and progress. 3rd ed. Philadelphia: Lippincott; c1997.

5. Souza AMA, Fraga MNO, Moraes LMP, Garcia MLP, Moura KDR, Almeida PC, Moura emv. Grupo terapêutico: sistematização da assistência de enfermagem em saúde mental. Texto \& Contexto Enfermagem. 2004; 13(4):625-32.

6. Taylor CM. Fundamentos de enfermagem psiquiátrica de Mereness. 13a ed. Porto Alegre: Artes Médicas; 1992.

7. Loomis ME. Group process for nurses. St. Louis: Mosby; 1979.

8. Munari DB, Furegato AR. Enfermagem e grupos. Goiânia: Ab; 1997.

9. Parker ME. Nursing theories and nursing practice. Philadelphia: F.A.Davis; 2000.

10. Moreira RVO, Pinheiro AKB, Moreira TMM, Fialho AVM. Relacionamento interpessoal em enfermagem: Habermas e Peplau. In: Barreto JAE, Moreira RVO, organizadores. A outra margem: filosofia, teorias de enfermagem e cuidado humano. Fortaleza: Casa José de Alencar / Programa Editorial; 2001. p. 205-25.

11. Ataíde MBC, Pagliuca LMF, Damasceno MMC. Inter-relação dos propósitos da teoria de Peplau com o cuidado ao diabético. Rev Bras Enfermagem. 2002; 55(6): 674-9.

12. George JB (ed.). Teorias de enfermagem: os fundamentos à prática profissional. 4. ed. Porto Alegre: Artmed; 2000.

13. Barnum BS. Nursing theory: analysis, application, evaluation. 5th ed. Philadelphia: Lippincott; c1998.

14. Pinheiro AKB. Depois do parto tudo muda: um novo olhar sobre adolescentes nutrizes. [tese]. Fortaleza: Departamento de Enfermagem da Faculdade de Farmácia, Odontologia e Enfermagem da Universidade Federal do Ceará; 2003. 